\title{
Exposure to polycyclic aromatic hydrocarbons in petrochemical industries by measurement of urinary 1-hydroxypyrene
}

\author{
P J Boogaard, N J van Sittert
}

\begin{abstract}
Biological monitoring of exposure of workers to polycyclic aromatic hydrocarbons (PAHs) in petrochemical industries was performed by the measurement of urinary excretion of 1-hydroxypyrene. In 121 of the 462 workers studied (both smokers and non-smokers) who had had no recent occupational exposure to PAHs a median 1-hydroxypyrene concentration of $0.21 \mu \mathrm{g} / \mathrm{g}$ creatinine was found. The upper limit of the $95 \%$ confidence interval in these workers of $0.99 \mu \mathrm{g} / \mathrm{g}$ creatinine was used as the upper normal value for industrial workers. Urinary 1-hydroxypyrene concentrations were measured in workers involved in manufacture and maintenance operations in oil refineries (13 studies in eight different settings), in workers manufacturing or handling products containing PAHs in chemical plants (five studies in three settings) and laboratories (four studies), and in workers digging soil contaminated with PAHs (three studies). In most studies in oil refineries 1-hydroxypyrene concentrations were only marginally greater than the values measured in the 121 workers with no recent occupational exposure to PAHs. This was also the case in maintenance operations with higher potential exposure to PAHs, indicating that personal protection equipment was generally adequate to prevent excessive exposure. The studies in chemical plants also showed that exposure to PAHs is low. An exception was the workers engaged in the production of needle coke from ethylene cracker residue, where increased urinary 1-hydroxypyrene concentrations were measured. The excretion of 1-hydroxypyrene by the operators and maintenance workers of this plant was investigated in relation to potential methods of exposure to PAHs. Dermal and inhalatory exposure were both significant determinants of exposure to PAHs. Dermal absorption contributed more to the overall exposure to PAHs in maintenance workers than in operators.
\end{abstract}

(Occup Environ Med 1994;51:250-258)

Polycyclic aromatic hydrocarbons (PAHs) are ubiquitous in our environment as they may be formed during any incomplete combustion of organic material. Some PAHs with four or more condensed benzene rings are suspected to be human carcinogens. ${ }^{1}$ As a consequence, exposure to PAHs may present a health risk in several industrial settings such as chemical manufacturing plants and oil refineries. Exposure to PAHs is in virtually all cases a combined exposure because they are always present in complex mixtures of up to 100 or more different PAHs. This implies that monitoring of these compounds, either by ambient or biological monitoring, is difficult. Therefore, a suitable marker representative of the complete set of PAHs has to be used and because only PAHs with four or more rings are carcinogenic, one of the larger PAHs should be selected for the purpose.

In general, PAHs are extensively metabolised to many different (poly-)hydroxyPAHs. These metabolites are mainly excreted in the faeces as conjugates and only a minor fraction is excreted in the urine. The four ring PAH pyrene, however, is an exception as it is almost exclusively metabolised to 1 -hydroxypyrene, which accounts for about $90 \%$ of the total urinary excretion of pyrene in all species studied so far, including humans. ${ }^{2-7}$ The abundance of pyrene is always relatively high in PAH mixtures, ${ }^{7-10}$ and after its introduction by Jongeneelen et $a^{11}$ in 1985, 1-hydroxypyrene has been used as a variable for the biological monitoring of exposure to $\mathrm{PAH}$ in various studies. These studies involved workers engaged in the manufacture of graphite electrodes, ${ }^{910}$ coke oven workers, ${ }^{10-14}$ and people exposed to coal $\operatorname{tar}^{1114-20}$ and creosote. ${ }^{11,19}$ It was shown that the urinary excretion of 1-hydroxypyrene correlates well with the urinary excretion of mutagens. ${ }^{414}$ Measurement of urinary 1-hydroxypyrene was both more sensitive and more specific than the measurement of thioether excretion ${ }^{16}$ and urinary mutagenicity. ${ }^{17}$

As yet, no data on the actual exposure to PAHs in chemical manufacturing plants and oil refineries have been reported. In our report we discuss the results of biological monitoring of exposure to PAHs by measuring urinary 1-hydroxypyrene in several settings in the petrochemical industry. Particular attention was given to workers producing needle coke from ethylene cracker residue. We investigated the excretion of 1-hydroxypyrene by these workers in relation to potential methods of exposure to PAHs. 
Materials and methods

STUDY POPULATIONS AND URINE COLIECTION To evaluate the exposure to PAHs under various circumstances in the petrochemical industry, urinary 1-hydroxypyrene was measured in a series of studies in various settings in The Netherlands from 1987 to 1992 . The studies have been carried out on workers involved in manufacturing and maintenance operations in refineries (studies $1-8$ ), in the handling or manufacture of products containing PAHs in chemical plants (studies 9-11) and in laboratories (study 12), and on men digging soil contaminated with PAHs (study 13). If not mentioned otherwise, urine samples were collected in polyethene containers at the beginning of the first day of the shift (blank samples) and at the end of every working day during the shift (post-shift samples), and stored frozen at $-20^{\circ} \mathrm{C}$ until analysis. In some studies baseline values for industrial populations were determined in urine samples collected after a period (at least three days) in which no occupational exposure had occurred.

Studies $1 \mathrm{a}$ and $1 \mathrm{~b}$ were performed on 22 men in 1987 and 17 men in 1992 involved in a clean out operation of steam boilers fired with fuel oil residue. The men wore PVC gloves and occasionally used supplied air line respirators to protect themselves from dusts containing PAHs during the removal of boiler linings.

Study 2 was performed in 1987 on five workers involved in an inspection operation of a furnace of a crude oil distillation unit. The men wore PVC gloves and half-mask respirators with particulate filter cartridges.

Study 3 took place in 1989 and involved four operators loading bitumen road tankers. Apart from occasional use of PVC gloves no personal protection was applied. As these four operators had had no recent potential exposure to PAHs, their pre-exposure samples could serve as baseline values (baseline study 3 ).

Three studies were carried out during cleaning out platforming installations in 1989 (study 4a, 14 men) and 1991 (studies 4b, 14 men; and $4 \mathrm{c}$ five men). During the various operations PVC gloves were used and also half-mask respirators with particulate filter cartridges.

Study 5 was performed on 24 contractor personnel during the cleaning out of a hydroconverter installation in 1990. The men wore PVC gloves and, dependent on the type of work they carried out, half-mask respirators with particulate filter cartridges or full facepiece respirators with an air line.

Study 6 was carried out in 1990 on three operators handling furfural extracts contaminated with PAHs. The operators wore PVC gloves and boots.

Three studies were carried out on contractor personnel involved in cleaning tanks in which liquids containing PAHs had been stored. Study 7 a was in 1990 on 24 men wearing PVC gloves and fresh air hoods. Study $7 \mathrm{~b}$ was in 1991 on 12 men and $7 \mathrm{c}$ was in 1992 on 13 men. The men working inside the tank were protected by fully encapsulating suits with an air line. The men outside the tanks wore PVC gloves and half-face respirators with organic vapour cartridges. The workers from study 7c had no recent potential occupational exposure to PAHs and their blank samples were used as controls (baseline study 7c).

Study 8 was carried out in 1991 on six people involved in cleaning out a hydrodesulphurisation installation. The personnel inside the installations wore a fully encapsulating PVC suit with supplied air line and the men outside the reactors were protected by PVC gloves and boots and full facepiece respirators with an airline.

Study 9 was performed in 1987 on four maintenance workers replacing burners in boilers. Half-mask respirators with particulate filter cartridges and PVC gloves were used during the work. This work took several weeks during which the four workers provided a series of blank samples (baseline study 9).

Study 10 was performed in 1991 on seven operators producing rubber grades containing aromatic extracts. Apart from PVC gloves no personal protection was used.

Study 11a was performed in 1988 on 57 people, $11 \mathrm{~b}$ in 1990 on 28 people, and $11 \mathrm{c}$ in 1991 on 25 people, involved in operational and maintenance activities at a needle coke plant. Cotton and PVC gloves were worn and, in 1991, occasionally half-mask respirators with particulate filter cartridges.

Four studies were carried out on laboratory personnel handling clarified slurry oils; study $12 \mathrm{a}$ in 1987 on 27 workers, studies $12 \mathrm{~b}$ and 12c were in 1988 on 11 and four workers, and study 12d was in 1989 on one worker. The workers handling the oils wore latex gloves. Two control studies were performed in 1987 and 1988 on 15 and 11 persons from the laboratory who had had no recent exposure to materials containing PAHs (baseline studies $12 \mathrm{a}$ and $12 \mathrm{~b}$ ).

Three studies, 13a in $1987 / 8$ on 62 men, $13 \mathrm{~b}$ in 1991 on seven men, and $13 \mathrm{c}$ in 1992 on four men, were performed on contractor personnel digging in soil contaminated with PAHs. In 1987-8 the men wore half-mask respirators with organic vapour cartridges only occasionally. In 1991 and 1992 halfmask respirators with organic vapour cartridges and PVC suits were worn throughout the study. As the workers from studies $13 \mathrm{~b}$ and $13 \mathrm{c}$ had no recent occupational exposure to PAHs, their pre-exposure samples could serve as control samples (baseline studies 13b and $13 \mathrm{c}$ ).

Finally, in 1989 a study was performed on 63 workers of a bitumen production plant at the end of a shut down period in which no occupational exposure to PAHs could have occurred (baseline study 14).

If more than one blank sample (baseline study 9) or post-shift sample (studies 1-4 and 6-13) was collected from a worker the 1-hydroxypyrene results were averaged. The 
Table 1 Baseline (pre-exposure) values of urinary 1-hydroxypyrene concentrations ( $\mu \mathrm{g} / \mathrm{g}$ creatinine) in various industrial populations

\begin{tabular}{|c|c|c|c|c|c|}
\hline \multirow{2}{*}{$\begin{array}{l}\text { Baseline } \\
\text { study }\end{array}$} & \multirow[b]{2}{*}{ Activity } & \multirow{2}{*}{$\begin{array}{l}\text { No of } \\
\text { workers } \\
\text { (samples) }\end{array}$} & \multicolumn{3}{|c|}{$\begin{array}{l}\text { 1-Hydroxypyrene } \\
\text { ( } \mu \text { g/g creatinine })\end{array}$} \\
\hline & & & Median & Mean & Range \\
\hline $\begin{array}{l}3 \\
7 \mathrm{c} \\
9 \\
12 \mathrm{a} \\
12 \mathrm{~b} \\
13 \mathrm{~b} \\
13 \mathrm{c} \\
14 \\
\text { Total }\end{array}$ & $\begin{array}{l}\text { Bitumen road tanker loading } \\
\text { Tank cleaningt } \\
\text { Boiler operation } \\
\text { Handling of clarified slurry oil } \\
\text { (Laboratory workers) } \\
\text { Digging† } \\
\text { Digging } \\
\text { Bitumen manufacturing } \ddagger\end{array}$ & $\begin{array}{l}4(4) \\
13(13) \\
4(28) \\
15(15) \\
11(11) \\
7(7) \\
4(4) \\
63(63) \\
121(145)\end{array}$ & $\begin{array}{l}0 \cdot 20 \\
0 \cdot 30 \\
0 \cdot 28 \\
0 \cdot 24 \\
0 \cdot 21 \\
0 \cdot 37 \\
0 \cdot 43 \\
0 \cdot 17 \\
0 \cdot 21\end{array}$ & $\begin{array}{l}0 \cdot 23 \\
0 \cdot 37 \\
0 \cdot 27 \\
0 \cdot 49 \\
0 \cdot 37 \\
0 \cdot 42 \\
0 \cdot 48 \\
0 \cdot 24 \\
0 \cdot 32\end{array}$ & 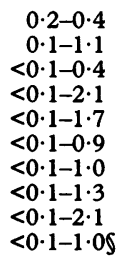 \\
\hline $\begin{array}{l}\text { Tolos et al } \\
\text { Jongeneelen et al } \\
\\
\text { Jongeneelen et } a l^{18}\end{array}$ & $\begin{array}{l}\text { Aluminium smelting } \\
\text { Coking (non-smokers) } \\
\text { Coking (smokers) } \\
\text { Paving workers }\end{array}$ & $\begin{array}{l}23(23) \\
19(19) \\
25(25) \\
16(43)\end{array}$ & $\begin{array}{l}1.49 \\
1.90\end{array}$ & 0.85 & $\begin{array}{l}0 \cdot 2-1 \cdot 1 \\
0 \cdot 5-3 \cdot 3 \\
0 \cdot 5-6 \cdot 8\end{array}$ \\
\hline
\end{tabular}

^No occupational exposure to PAH for 2-3 days.

†No occupational exposure to PAH for at least 3 days.

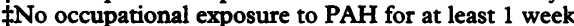

SLimits of the $95 \% \mathrm{CI}$.
(Breda, The Netherlands). Solid samples of the produced coke and of the residue remaining on the litters of the coke chambers were analysed for PAH content by TNO laboratories (Delft, The Netherlands).

\section{Work registration}

Each day, at the end of the shift, the workers completed a questionnaire on working conditions, specific activities during the shift, the use of personal protective devices, and smoking behaviour. An industrial hygienist made a daily, semiquantitative assessment of the extent of potential inhalatory and of dermal exposure by ranking the extent of steam formation and the temperature of the liberated steam during the opening of the coke chambers and the amount of coke remaining on the litter $(0=$ less than average; $1=$ average; $2=$ more than average; $3=$ much more than average).

median and mean for the group of workers were then calculated from the individually averaged results.

\section{NEEDLE COKE PLANT STUDY}

In study 11a high urinary concentrations of 1-hydroxypyrene were measured in workers employed at a needle coke plant during normal operation. It was suggested that the highest risk of exposure was present during the opening of the coke chambers, when steam contaminated with PAHs is released, and during maintenance. To find out what kind of measures had to be taken to reduce exposure a more detailed study was performed in 1990.

Study populations and collection of urine samples This more elaborate study comprised 25 men working in a five shift system either as operator (12 men) or as maintenance personnel (13 men). The workers were monitored during five consecutive night shifts in 1990. Smoking behaviour was not recorded from three men; 11 out of the remaining 22 men were smokers. There was no significant difference in smoking behaviour (yes/no) between operators and maintenance personnel ( $p=0.395$, Fisher's exact test).

Operators and maintenance personnel were asked to collect pre- and post-shift urine samples on each day of the shift (five consecutive nights). The first pre-shift sample, collected after three days leave, was considered to represent a blank sample.

\section{Air monitoring}

Air (both static and personal) was sampled by National Institute of Occupational Safety and Health (NIOSH) method 5515 with XAD-2 tubes and analysed by gas liquid chromatography with flame ionisation detection. A second set of air samples (static) was collected according to the Dutch standardised methods NVN 2798 and 2816 during the opening of the coke chambers and analysed by high performance liquid chromatography with UV detection at BCO laboratories

\section{Calculations and statistics}

The results for the urinary 1-hydroxypyrene concentrations before and after shifts were averaged for each worker and the group median and mean were calculated from these averaged results. From the decline in individual urinary 1-hydroxypyrene during the night (the difference between the concentration in the post-shift sample $\left(c_{\mathrm{e}}\right)$ collected at the end of the shift $\left(t_{e}\right)$ and the concentration in the pre-shift sample $\left(\mathrm{c}_{\mathrm{b}}\right)$ collected the next morning $\left(t_{b}\right)$ ) the urinary elimination rate constant $\left(k_{e}\right)$ of 1-hydroxypyrene was calculated with a one compartment kinetic model assuming completed absorption and first order elimination using the formula:

$$
\ln \left(c_{b} / c_{e}\right)=-k_{e} \cdot\left(t_{b}-t_{e}\right)
$$

Urinary excretion half lives were only calculated from urinary samples provided by workers who had had a distinct exposure to PAHs (with a 1-hydroxypyrene concentration in the post-shift sample of at least the upper limit of the $95 \%$ confidence interval (95\% CI) for baseline values in industrial workers). Forty two sets of urine samples, collected from 16 of the 25 persons, met this criterion and were used to calculate $a k_{e}$. If $k_{e}$ could be calculated more than once for a worker the data were averaged. From the median $k_{e}$ the corresponding median apparent half life of elimination $\left(t_{1 / 2}\right)$ was calculated from the formula

$$
t_{1 / 2}=\ln (2) / k_{e} \text {. }
$$

The strength of three possible methods of exposure (smoking habits, inhalatory, and dermal exposure) in relation to the urinary concentrations of 1-hydroxypyrene was studied by multiple linear regression analysis after normalisation of the distribution by logarithmic transformation. The $\mathbf{R}^{2}$ values and the $p$ values of the $F$ test are tabulated.

For all statistical calculations the mainframe version of the SAS statistical software package (version 6.06) was used and p $<0.05$ was considered significant. 
Table 2 Published data on urinary 1-hydroxypyrene concentrations (in $\mu g / g$ creatinine) in people not occupationally exposed

\begin{tabular}{|c|c|c|c|c|c|c|}
\hline \multirow[b]{2}{*}{ Baseline study } & \multirow[b]{2}{*}{ Group } & & \multirow{2}{*}{$\begin{array}{l}\text { No of } \\
\text { workers } \\
\text { (samples) }\end{array}$} & \multicolumn{2}{|c|}{$\begin{array}{l}\text { 1-Hydroxypyrene } \\
\text { ( } \mu \mathrm{g} / \mathrm{g} \text { creatinine })\end{array}$} & \multirow[b]{2}{*}{ Range } \\
\hline & & & & Median & Mean & \\
\hline Buchet et al ${ }^{10}$ & Belgian males & $\begin{array}{l}(\mathbf{N}) \\
(\mathbf{S})\end{array}$ & $\begin{array}{l}9(9) \\
6(6)\end{array}$ & $\begin{array}{l}0.15 \\
0.32\end{array}$ & - & - \\
\hline Jongeneelen et al ${ }^{11} 20$ & Dutch males & $\begin{array}{l}\text { (N) } \\
\text { (S) }\end{array}$ & $\begin{array}{l}52(52) \\
38(38)\end{array}$ & $\begin{array}{l}0.52 \\
0.56\end{array}$ & 二 & $\begin{array}{l}<0 \cdot 1-1 \cdot 6 \\
<0 \cdot 1-2 \cdot 8\end{array}$ \\
\hline Jongeneelen et al ${ }^{12}$ & Dutch males & $\begin{array}{l}\text { (N) } \\
\text { (S) }\end{array}$ & $\begin{array}{l}14(14) \\
28(28)\end{array}$ & $\begin{array}{l}0.33 \\
0.99\end{array}$ & - & $\begin{array}{r}<0 \cdot 1-1 \cdot 8 \\
0 \cdot 1-2 \cdot 7\end{array}$ \\
\hline Jongeneelen et al ${ }^{15}$ & Dutch males & $\begin{array}{l}\text { (N) } \\
(\mathbf{S})\end{array}$ & $\begin{array}{l}24(24) \\
22(22)\end{array}$ & $\begin{array}{l}0.45 \\
0.52\end{array}$ & - & $\begin{array}{l}0 \cdot 1-1 \cdot 7^{\star} \\
0 \cdot 12-2 \cdot 2^{\star}\end{array}$ \\
\hline Zhao et al ${ }^{13}$ & $\begin{array}{l}\text { Anshan males } \\
\text { Shenyang females } \\
\text { Taiyuan females } \\
\text { Beijing females } \\
\text { Beijing girls }\end{array}$ & $\begin{array}{l}\text { (S) } \\
\text { (N) } \\
\text { (N) } \\
\text { (N) }\end{array}$ & $\begin{array}{l}10(10) \\
13(13) \\
17(17) \\
15(15) \\
15(15)\end{array}$ & $\begin{array}{l}0.6 \\
1.7 \\
1.6 \\
0.8 \\
1.1\end{array}$ & $\begin{array}{l}0.6 \\
1.9 \\
1.4 \\
0.7 \\
0.9\end{array}$ & $\begin{array}{l}0 \cdot 2-0 \cdot 9 \\
1 \cdot 5-3 \cdot 4 \\
0 \cdot 6-2 \cdot 4 \\
0 \cdot 2-1 \cdot 6 \\
0 \cdot 2-1 \cdot 7\end{array}$ \\
\hline Zhao et al ${ }^{22}$ & Beijing males & $\begin{array}{l}\text { (N) } \\
\text { (S) }\end{array}$ & $\begin{array}{l}74(74) \\
84(84)\end{array}$ & - & $\begin{array}{l}1 \cdot 31 \\
1 \cdot 47\end{array}$ & - \\
\hline & $\begin{array}{l}\text { Beijing males } \\
\text { Beijing females }\end{array}$ & & $\begin{array}{c}121(121) \\
30(30)\end{array}$ & - & $\begin{array}{l}1.26 \\
1.18\end{array}$ & 二 \\
\hline Burgas et al ${ }^{16}$ & Turkish males & $\begin{array}{l}\text { (N) } \\
\text { (S) }\end{array}$ & $\begin{array}{l}15(15) \\
14(14)\end{array}$ & - & $\begin{array}{l}0.47 \\
0.64\end{array}$ & - \\
\hline
\end{tabular}

*Limits of the $95 \% \mathrm{CI}$.

$\mathrm{S}=$ smokers; $\mathrm{N}=$ non-smokers. homogeneity. A $5 \mathrm{ml}$ aliquot of the sample was adjusted to $\mathrm{pH} 5.0$ and $2.5 \mathrm{ml}$ sodium acetate buffer $\mathrm{pH}=5.0$ and $2500 \mathrm{U} \beta$-glucuronidase/aryl sulphatase (Boehringer Mannheim, Germany) were added. The mixture was incubated overnight in a rotary shaker water bath at $37^{\circ} \mathrm{C}$ and subsequently run slowly through a reversed phase $\mathrm{C} 18$ Bond-Elut extraction cartridge. The cartridge was washed with purified water and the extracted 1-hydroxypyrene was then eluted with $10 \mathrm{ml}$ methanol. The organic extract was evaporated at $60^{\circ} \mathrm{C}$ under a gentle flow of nitrogen until dry and the residue was reconstituted with $1.0 \mathrm{ml}$ methanol by sonification to effect complete dissolution. After centrifugation of the reconstituted extract the clear supernatant liquid was transferred into a high performance liquid chromatography (HPLC) autosampler vial.

The samples were analysed with a reversed phase HPLC system equipped with a Waters $600 \mathrm{E}$ pulse free pump system and a WISP 712 autosampler. The system was equipped with a Perkin Elmer LS40 fluorescence detector (excitation wavelength $242 \mathrm{~nm}$ and emission wavelength $388 \mathrm{~nm}$ ) and fitted with a $150 \times 4.6 \mathrm{~mm}$ (internal diameter) stainless steel column packed with $5 \mu \mathrm{m}$ Lichrosorb RP18 (Chrompack, The Netherlands) and

Table 3 Urinary 1-hydroxypyrene concentrations ( $\mu g / g$ creatinine) in workers engaged in various industrial settings

\begin{tabular}{|c|c|c|c|c|c|c|c|c|c|}
\hline \multirow{2}{*}{$\begin{array}{l}\text { Study } \\
\text { No }\end{array}$} & \multirow{2}{*}{ Description } & \multirow[b]{2}{*}{ Year } & \multirow{2}{*}{$\begin{array}{l}\text { No of } \\
\text { workers } \\
\text { (samples) }\end{array}$} & \multicolumn{3}{|c|}{$\begin{array}{l}\text { 1-Hydroxypyrene } \\
\text { ( } \mu \text { glg creatinine })\end{array}$} & \multirow{2}{*}{$\begin{array}{l}\text { Total PAHs } \\
(\geqslant 3 \text { rings })\end{array}$} & \multirow{2}{*}{$\begin{array}{l}\text { Pyrene as } \% \\
\text { of total PAHs } \\
(\geqslant 3 \text { rings })\end{array}$} & \multirow{2}{*}{$\begin{array}{l}\text { Ratio pyrene: } \\
\text { benzo (a)pyrene }\end{array}$} \\
\hline & & & & Median & Mean & Range & & & \\
\hline $\begin{array}{l}\text { Oil ref } \\
\text { la } \\
\text { lb }\end{array}$ & $\begin{array}{l}\text { finery: } \\
\text { Cleaning steam boiler }\end{array}$ & $\begin{array}{l}1987 \\
1992\end{array}$ & $\begin{array}{l}22(98) \\
17(40)\end{array}$ & $\begin{array}{l}0.68 \\
0.69\end{array}$ & $\begin{array}{l}0.51 \\
0.82\end{array}$ & $\begin{array}{r}<0 \cdot 1-6 \cdot 8 \\
0 \cdot 2-3 \cdot 2\end{array}$ & $<0.3 \dagger$ & * & - \\
\hline 2 & $\begin{array}{l}\text { Inspecting furnace of a } \\
\text { crude distillation unit }\end{array}$ & 1987 & $5(25)$ & 1.56 & 3.03 & $0.7-8.5$ & $2 \cdot 3-5 \cdot 8 \dagger$ & $<3 \dagger$ & - \\
\hline 3 & Loading bitumen road tanker & 1989 & $4(25)$ & 0.38 & 0.43 & $0.1-0.8$ & $3 \cdot 9-23 t$ & $4-7 \dagger$ & $0.4-1 \cdot 2 \dagger$ \\
\hline $\begin{array}{l}4 a \\
4 b \\
4 c\end{array}$ & $\begin{array}{l}\text { Cleaning platforming } \\
\text { installations }\end{array}$ & $\begin{array}{l}1989 \\
1991 \\
1991\end{array}$ & $\begin{array}{r}14(95) \\
14(75) \\
5(26)\end{array}$ & $\begin{array}{l}0.45 \\
0.62 \\
0.25\end{array}$ & $\begin{array}{l}0.61 \\
0.62 \\
0.29\end{array}$ & $\begin{array}{r}<0 \cdot 1-2 \cdot 1 \\
0 \cdot 2-2 \cdot 7 \\
<0 \cdot 1-0.9\end{array}$ & $\overline{\bar{z}}$ & $\begin{array}{l}\bar{\star} \\
11-34 \dagger\end{array}$ & $\overline{\bar{c}}-170+$ \\
\hline 5 & Cleaning hydroconverter & 1990 & $24(24)$ & 0.52 & 0.63 & $0 \cdot 15-1 \cdot 9$ & $0.02-0.67 \dagger$ & $10-18 \dagger$ & $30-220 t$ \\
\hline 6 & Handling furfural extract & 1990 & $3(5)$ & 0.40 & 0.40 & $0.2-0.5$ & - & - & - \\
\hline $\begin{array}{l}7 \mathrm{a} \\
7 \mathrm{~b} \\
7 \mathrm{c}\end{array}$ & Cleaning tanks & $\begin{array}{l}1990 \\
1991 \\
1992\end{array}$ & $\begin{array}{l}24(99) \\
12(51) \\
13(34)\end{array}$ & $\begin{array}{l}0 \cdot 48 \\
0 \cdot 50 \\
0 \cdot 43\end{array}$ & $\begin{array}{l}0.60 \\
0.63 \\
0.47\end{array}$ & $\begin{array}{l}<0 \cdot 1-6 \cdot 5 \\
<0 \cdot 1-2 \cdot 2 \\
<0 \cdot 1-1 \cdot 2\end{array}$ & $\bar{z}$ & $\bar{E}$ & $\bar{E}$ \\
\hline 8 & Cleaning desulphurisation unit & 1991 & $6(29)$ & 0.50 & 0.91 & $0 \cdot 2-2 \cdot 5$ & - & - & 一 \\
\hline \multicolumn{10}{|c|}{ Chemical plants: } \\
\hline 10 & $\begin{array}{l}\text { Manufacture of rubber grades } \\
\text { containing aromatic extracts }\end{array}$ & 1991 & $7(8)$ & $1 \cdot 1$ & 0.98 & $0 \cdot 3-1 \cdot 7$ & $\begin{array}{l}126-425 \dagger \\
<0.08 \ddagger\end{array}$ & $\begin{array}{l}9-13 \dagger \\
2-9 \ddagger\end{array}$ & $\begin{array}{l}>4 \cdot 5 \dagger \\
>6 \cdot 7 \ddagger\end{array}$ \\
\hline $\begin{array}{l}\text { Needle } \\
11 \mathrm{a}\end{array}$ & $\begin{array}{l}\text { le coke manufacturing: } \\
\text { Maintenance } \\
\text { Operation }\end{array}$ & 1988 & $\begin{array}{l}18(91) \\
39(231)\end{array}$ & $\begin{array}{l}2 \cdot 76 \\
1 \cdot 18\end{array}$ & $\begin{array}{l}3 \cdot 74 \\
2 \cdot 16\end{array}$ & $\begin{array}{r}0.4-18.5 \\
<0.1-45.4\end{array}$ & - & - & - \\
\hline $\begin{array}{l}11 \mathrm{~b} \\
11 \mathrm{c}\end{array}$ & $\begin{array}{l}\text { Maintenance } \\
\text { Operation } \\
\text { Maintenance } \\
\text { Operation } \\
\text { Contractors }\end{array}$ & $\begin{array}{l}1990 \\
1991\end{array}$ & $\begin{array}{c}13(105) \\
15(101) \\
4(21) \\
13(44) \\
8(44)\end{array}$ & $\begin{array}{l}1.63 \\
1.65 \\
1.51 \\
0.63 \\
1.30\end{array}$ & $\begin{array}{l}1.91 \\
3.93 \\
1.69 \\
0.86 \\
1.74\end{array}$ & $\begin{array}{l}0 \cdot 3-5 \cdot 5 \\
0 \cdot 3-25 \cdot 5 \\
0 \cdot 3-4 \cdot 9 \\
0 \cdot 1-3 \cdot 2 \\
0 \cdot 1-9 \cdot 4\end{array}$ & $\begin{array}{l}0 \cdot 6 \dagger \\
0 \cdot 1-30 \ddagger \\
0 \cdot 03-0.08 \dagger\end{array}$ & $\begin{array}{l}16 \dagger \\
7-26 \ddagger \\
11-18 \dagger\end{array}$ & $\begin{array}{r}2 \cdot 4 \dagger \\
>16 \cdot 4 \ddagger \\
6-12 \dagger\end{array}$ \\
\hline $\begin{array}{l}\text { Miscel } \\
12 \mathrm{a} \\
12 \mathrm{~b} \\
12 \mathrm{c} \\
12 \mathrm{~d}^{9}\end{array}$ & $\begin{array}{l}\text { Haneous: } \\
\text { Laboratory of clarified slurry oil } \\
\text { Labers }\end{array}$ & $\begin{array}{l}1987 \\
1988 \\
1988 \\
1989\end{array}$ & $\begin{array}{c}27(27) \\
11(11) \\
4(8) \\
1(6)\end{array}$ & $\begin{array}{l}0 \cdot 21 \\
0 \cdot 14 \\
0 \cdot 75 \\
4 \cdot 1\end{array}$ & $\begin{array}{l}0.40 \\
0.26 \\
1 \cdot 78 \\
5.9\end{array}$ & $\begin{aligned}< & 0.1-2.1 \\
< & 0.1-0.7 \\
& 0.5-5 \cdot 1 \\
& 1.0-9.6\end{aligned}$ & $\begin{array}{l}- \\
-\end{array}$ & $\begin{array}{l}z \\
-\end{array}$ & $\begin{array}{l}- \\
-\end{array}$ \\
\hline $\begin{array}{l}13 a \\
13 b\end{array}$ & $\begin{array}{l}\text { Digging soil contaminated } \\
\text { with PAHs }\end{array}$ & $\begin{array}{l}1987 / 8 \\
1991\end{array}$ & $\begin{array}{c}862(615) \\
7(27)\end{array}$ & $\begin{array}{l}0.97 \\
0.69\end{array}$ & $\begin{array}{l}1.56 \\
0.67\end{array}$ & $\begin{array}{l}<0 \cdot 1-32 \cdot 6 \\
<0 \cdot 1-2 \cdot 5\end{array}$ & $\begin{array}{l}0 \cdot 07 \dagger \\
23-1125\end{array}$ & $13 \dagger$ & $\begin{array}{c}12 \cdot 3 \dagger \\
5-225\end{array}$ \\
\hline $13 c$ & & 1992 & $4(40)$ & 0.43 & 0.48 & $<0.1-1.0$ & $2-969$ & $6-189$ & $4-24 \S$ \\
\hline
\end{tabular}

^Pyrene below level of quantitation.

†Solid samples (dust, product, or residue); PAHs in $\mathrm{mg} / \mathrm{kg}$.

Air samples; PAHs in $\mu \mathrm{g} / \mathrm{m}^{3}$.

SLiquid samples (ground water); PAHs in $\mu \mathrm{g} / 1$.

१Study 12d: one worker from Study $12 \mathrm{c}$. 
Table 4 Published data on urinary 1-hydroxypyrene concentrations ( $\mu g / g$ creatinine) in patients and in industrial populations exposed to PAHs

\begin{tabular}{|c|c|c|c|c|c|c|c|c|}
\hline \multirow{2}{*}{$\begin{array}{l}\text { Reference } \\
\text { No }\end{array}$} & \multirow[b]{2}{*}{ Activity } & \multirow{2}{*}{$\begin{array}{l}\text { No of } \\
\text { workers } \\
\text { (samples) }\end{array}$} & \multicolumn{3}{|c|}{$\begin{array}{l}\text { 1-Hydroxypyrene } \\
\text { ( } \mu \text { glg creatinine) }\end{array}$} & \multirow{2}{*}{$\begin{array}{l}\text { Total PAHs } \\
(\geqslant 3 \text { rings })\end{array}$} & \multirow{2}{*}{$\begin{array}{l}\text { Pyrene as } \% \\
\text { of total PAHs } \\
\text { ( } \geqslant 3 \text { rings })\end{array}$} & \multirow{2}{*}{$\begin{array}{l}\text { Ratio pyrene: } \\
\text { benzo(a)pyrene }\end{array}$} \\
\hline & & & Median & Mean & Range & & & \\
\hline \multirow{5}{*}{$\begin{array}{l}9 \\
4,15 \\
14 \\
15,17 \\
15,17\end{array}$} & Aluminium reduction & $28(54)$ & - & 4.93 & - & $28 \cdot 3+$ & $10 \cdot 0^{+}$ & - \\
\hline & Distillation of coal tar & $4(12)$ & $8 \cdot 3$ & $11 \cdot 7$ & $2 \cdot 3-25$ & $15 \cdot 2 \dagger$ & $33^{+}$ & $11^{\star}$ \\
\hline & Medical treatment with coal tar & $5(32)$ & - & - & $100-1000$ & - & - & - \\
\hline & (pix lithanthracis) & $\begin{array}{l}1(22) \\
3(8)\end{array}$ & - & $\overline{-}$ & $\begin{array}{l}25 \cdot 4-39 \cdot 2 \\
35 \cdot 1-1565\end{array}$ & 5 & - & $\begin{array}{l}2 \cdot 39^{\star} \\
2 \cdot 39^{\star}\end{array}$ \\
\hline & Coking: & & & & & & & \\
\hline \multirow[t]{2}{*}{10} & Belgium, topside of oven: & & & & & $131 \dagger$ & $7 \cdot 2 \dagger$ & $1 \cdot 75 \dagger$ \\
\hline & $S$ & $3(3)$ & $11 \cdot 7$ & - & - & - & - & - \\
\hline \multirow{3}{*}{10} & $\mathrm{~N}$ & $3(3)$ & $10 \cdot 9$ & - & - & $\overline{9} \cdot 3 t$ & $\overline{0.8+}$ & - $1.61+$ \\
\hline & $\mathrm{S}$ & $6(4)$ & 1.5 & - & - & - & - & $-1.01 \mathrm{~T}$ \\
\hline & $\mathbf{N}$ & $4(4)$ & $2 \cdot 4$ & - & - & - & - & - \\
\hline \multirow[t]{2}{*}{12} & The Netherlands: & & & & & $12.9 \dagger$ & $12 \cdot 4 \dagger$ & $1.6 \dagger$ \\
\hline & $\begin{array}{l}\mathbf{S} \\
\mathbf{N}\end{array}$ & $\begin{array}{l}25(25) \\
22(22)\end{array}$ & $\begin{array}{l}6 \cdot 5 \\
3 \cdot 7\end{array}$ & 二 & $\begin{array}{l}1 \cdot 1-14 \cdot 2 \\
0 \cdot 8-21 \cdot 7\end{array}$ & - & - & - \\
\hline 13 & China (Anshan plant) & $15(15)$ & $(36 \cdot 5)$ & $40 \cdot 0$ & $6 \cdot 8-120$ & - & - & - \\
\hline 13 & $\begin{array}{l}\text { China (Taiyuan plant) } \\
\text { Paving with asphalt: }\end{array}$ & $31(31)$ & $(16 \cdot 0)$ & $18 \cdot 4$ & $1 \cdot 1-57 \cdot 4$ & - & $10 \cdot 6 \dagger$ & $1 \cdot 79 \dagger$ \\
\hline \multirow{6}{*}{$\begin{array}{l}18 \\
19 \\
16 \\
16 \\
19 \\
20\end{array}$} & Bitumen and coal tar blend & $31(43)$ & - & 3.4 & $0 \cdot 8-16 \cdot 5$ & - & - & $4^{*}, 9 \dagger$ \\
\hline & 然 & $28(28)$ & $\mathbf{5 \cdot 4}$ & $4 \cdot 2$ & $1 \cdot 7-6 \cdot 2$ & - & - & - \\
\hline & $\Rightarrow \quad \mathbf{S}$ & $21(21)$ & $1 \cdot 2$ & $1 \cdot 3$ & $0.3-2.6$ & - & - & - \\
\hline & $\ddot{N}$ & $18(18)$ & 0.8 & $\begin{array}{l}1.0 \\
1.2\end{array}$ & $0.4-3 \cdot 5$ & - & - & - \\
\hline & Wood impregnation & & & & & & & \\
\hline & with creosote & $3(47)$ & - & - & $2-39$ & $15 \cdot 2 \dagger$ & $33.4 \dagger$ & $11^{\star}$ \\
\hline 19 & $\begin{array}{l}\text { Gate-keeping: } \\
\text { (exposure to diesel exhausts): }\end{array}$ & $1(13)$ & & & $20-104$ & - & - & $3000^{n}$ \\
\hline 19 & $\stackrel{1}{\mathbf{S}}$ & $4(8)$ & - & $1 \cdot 3$ & - & - & - & - \\
\hline 19 & $\mathbf{N}$ & $3(6)$ & - & $0 \cdot 8$ & - & - & - & - \\
\hline
\end{tabular}

`Solid sample (dust, product or residue); PAH in $\mathrm{mg} / \mathrm{kg}$

tair samples; PAHs in $\mu \mathrm{g} / \mathrm{m}^{3}$.

$\mathrm{S}=$ smoker; $\mathbf{N}=$ non-smoker.

controlled at $40^{\circ} \mathrm{C}$ in an oven. A $20 \mu$ sample was applied on to the column through a $75 \times$ $2.1 \mathrm{~mm}$ (internal diameter) guard column packed with $30 \mu \mathrm{m} \mathrm{RP}$ material (Chrompack) and eluted with a constant flow of $0.8 \mathrm{ml} / \mathrm{min}$ with the following solvent programme: $5 \mathrm{~min}$ utes $55 / 45 \%(\mathrm{v} / \mathrm{v})$ methanol/water, then by a linear gradient to $98 / 2 \%(\mathrm{v} / \mathrm{v})$ methanol/ water in 30 minutes, back to $55 / 45 \%(v / v)$ methanol/water in 5 minutes, and stopped 5 minutes later. Methanol and water (both Baker HPLC grade) were degassed before and during sample runs by purging with helium $(30 \mathrm{ml} / \mathrm{min})$.

A series of urine samples selected from highly exposed workers from studies 1a, 11a, and $13 \mathrm{a}$ were pooled and aliquots were stored frozen at $-20^{\circ} \mathrm{C}$. In all runs a set of samples from this urine pool was analysed to check recovery and reproducibility of the method. The 1-hydroxypyrene concentration of the urine pool was 8.76 (SD 1.05$) \mu \mathrm{g} / \mathrm{g}$ creatinine. Over four years the urine pool was analysed regularly $(n=59)$, and the coefficient of variation was $12.0 \%$. The recovery was $87 \cdot 4(\operatorname{SD} 5 \cdot 8 \%)(n=26)$.

\section{Results}

\section{ROUTINE STUDIES}

Table 1 lists the baseline values of workers with potential occupational exposure as measured in the current studies. It seems (table 2) that background concentrations in people who perform jobs with potentially low exposure to PAHs, such as bitumen manufacturing, are, after a period of shut down, in the same range as found in Dutch people not

Table 5 PAH concentrations in air $\left(\mu \mathrm{g} / \mathrm{m}^{3}\right)$ in the needle coke plant study

\begin{tabular}{|c|c|c|c|c|c|c|}
\hline \multirow[b]{2}{*}{ Compound } & \multicolumn{3}{|c|}{ Static sampling (5 samples) } & \multicolumn{3}{|c|}{ Personal air sampling (7 samples) } \\
\hline & Median & Mean & Range & Median & Mean & Range \\
\hline $\begin{array}{l}\text { Acenaphthene } \\
\text { Acenaphthylene } \\
\text { Anthracene } \\
\text { Benz(a)anthracene/chrysene } \\
\text { Benzo(b)fluorene } \\
\text { Benzo(k)fluorene } \\
\text { Benzo(ghi)perylene } \\
\text { Benzo(a)pyrene } \\
\text { Dibenz(a,h)anthracene } \\
\text { Fluoranthene } \\
\text { Fluorene } \\
\text { Indeno(1,2,3-cd)pyrene } \\
\text { Naphthalene } \\
\text { Phenanthrene } \\
\text { Pyrene } \\
\text { Others }\end{array}$ & $\begin{array}{l}2 \cdot 2 \\
0.41 \\
0.53 \\
0 \cdot 13 \\
<0 \cdot 18 \\
<0.17 \\
<0.18 \\
<0.17 \\
<0.17 \\
1.01 \\
3.04 \\
<0 \cdot 17 \\
5 \cdot 10 \\
12.4 \\
1.50 \\
<2.5\end{array}$ & $\begin{aligned} & 3.3 \\
&<0.6 \\
& 0.66 \\
& 0.20 \\
&<0.21 \\
&<0.17 \\
&<0.21 \\
&<0.07 \\
&<0.25 \\
& 0.98 \\
& 4.93 \\
&<0.17 \\
& 5.32 \\
& 11.9 \\
& 1.71 \\
&<3.8\end{aligned}$ & $\begin{array}{l}1.3-8.0 \\
0.24-<1.8 \\
0.09-1.7 \\
0.08-0.3 \\
<0.17-<0.28 \\
<0.17-<0.18 \\
<0.17-<0.28 \\
<0.01-<0.22 \\
<0.17-<0.42 \\
0.30-1.63 \\
1.11-10.8 \\
<0.17-<0.18 \\
3.3-7.5 \\
1.84-25.0 \\
<0.17-3.55 \\
<0.01-<8.5\end{array}$ & $\begin{array}{r}0.02 \\
<0.01 \\
<0.01 \\
<0.01 \\
<0.01 \\
<0.01 \\
<0.01 \\
<0.01 \\
<0.01 \\
0.02 \\
<0.01 \\
<0.01 \\
0.11 \\
0.19 \\
0.04 \\
<0.01\end{array}$ & $\begin{array}{r}0.10 \\
0.06 \\
0.03 \\
0.05 \\
<0.01 \\
<0.01 \\
<0.01 \\
<0.01 \\
<0.01 \\
0.06 \\
0.06 \\
<0.01 \\
0.23 \\
0.24 \\
0.10 \\
<0.01\end{array}$ & $\begin{array}{r}<0.01-0.40 \\
<0.01-0.26 \\
<0.01-0.13 \\
<0.01-0.19 \\
<0.01-<0.01 \\
<0.01-<0.01 \\
<0.01-<0.01 \\
<0.01-<0.01 \\
<0.01-<0.01 \\
<0.01-0.21 \\
<0.01-0.23 \\
<0.01-<0.01 \\
0.02-0.79 \\
0.06-0.76 \\
<0.01-0.38 \\
<0.01-<0.01\end{array}$ \\
\hline Total benzene soluble matter & $29 \cdot 5$ & $24 \cdot 4$ & $\begin{array}{ll}4 & -42 \cdot 7\end{array}$ & 0.42 & 0.95 & $0.12-3.36$ \\
\hline Sampling rate $(1 / \mathrm{min})$ & $20 \cdot 0$ & $17 \cdot 6$ & $14 \cdot 0-20 \cdot 0$ & $32 \cdot 0$ & $32 \cdot 2$ & $8 \cdot 0-51 \cdot 3$ \\
\hline
\end{tabular}


Table 6 Typical composition of PAHs in coke samples: concentration of PAHs $(\mu \mathrm{g} / \mathrm{kg})$ in the needle coke plant study

\begin{tabular}{|c|c|c|c|}
\hline Compound & $\begin{array}{l}\text { Green BLS } \\
\text { coke } \\
\mu g / k g\end{array}$ & $\begin{array}{l}\text { Green } M L O \\
\text { coke } \\
\mu \mathrm{g} / \mathrm{kg}\end{array}$ & $\begin{array}{l}\text { Solid sample from } \\
\text { the litter of the } \\
\text { coke chamber } \\
\mu \mathrm{g} / \mathrm{kg}\end{array}$ \\
\hline $\begin{array}{l}\text { Acenaphthene } \\
\text { Acenaphthylene }\end{array}$ & - & - & $\begin{array}{l}8 \cdot 7 \\
7 \cdot 1\end{array}$ \\
\hline Antanthrene & 0.03 & $0 \cdot 190$ & - \\
\hline Anthracene & 0.54 & 1.7 & 23 \\
\hline Benz(a)anthracene & $1 \cdot 1$ & 3.5 & 9.9 \\
\hline Benzo(b)fluoranthene & 0.89 & $3 \cdot 3$ & - \\
\hline Benzo(j)fluoranthene & $<0.02$ & $<0.02$ & - \\
\hline Benzo(k)fluoranthene & $0 \cdot 13$ & 0.55 & - \\
\hline Benzo(b)fluorene & 0.65 & $2 \cdot 3$ & - \\
\hline Benzo(ghi)perylene & $0 \cdot 29$ & $1 \cdot 4$ & - \\
\hline Benzo(a)pyrene & 0.37 & 1.7 & 41 \\
\hline Benzo(e)pyrene & $<0.01$ & $<0.01$ & - \\
\hline Chrysene & $3 \cdot 3$ & 12 & 34 \\
\hline Dibenz $(\mathrm{a}, \mathrm{h})$ anthracene & 0.07 & 0.82 & - \\
\hline Dibenz $(a, j)$ anthracene & $<0.002$ & $0 \cdot 38$ & - \\
\hline Dibenzo(a,l)pyrene & $<0.002$ & $<0.002$ & - \\
\hline 1,4-Dimethylphenanthrene & $0 \cdot 23$ & 0.83 & - \\
\hline Fluoranthene & $1 \cdot 7$ & $4 \cdot 2$ & 43 \\
\hline Fluorene & - & - & 19 \\
\hline Indeno $(1,2,3$-cd)pyrene & $0 \cdot 15$ & 0.77 & - \\
\hline 3-Methylcholanthrene & 0.05 & 0.29 & - \\
\hline Naphthalene & - & - & 69 \\
\hline Perylene & $<0.006$ & 0.23 & - \\
\hline Phenanthrene & $4 \cdot 8$ & 17 & 255 \\
\hline Pyrene & $4 \cdot 5$ & $9 \cdot 3$ & 100 \\
\hline Triphenylene & 6.6 & 24 & - \\
\hline Total benzene soluble matter & $25 \cdot 4$ & $84 \cdot 5$ & $704 \cdot 8$ \\
\hline
\end{tabular}

occupationally exposed. As the variances in the results of the eight baseline studies were not statistically different (paired F test) the results were pooled and overall results were calculated. The overall median was $0.21 \mu \mathrm{g} / \mathrm{g}$ creatinine and the $95 \% \mathrm{CI}<0.1-0.99 \mu \mathrm{g} / \mathrm{g}$ creatinine. Data reported by other authors (tables 1 and 2) show that in operations where much higher occupational exposures to PAHs may occur (such as paving and coking) background concentrations are much higher and obviously do not return to normal during a relatively short period of non-exposure.

Table 3 summarises the results of the studies 1 to 13. In some studies the composition of the source of PAH exposure was quantitatively determined. In these cases, the amount of pyrene as a percentage of the total amount of PAHs with three or more rings have been included in tables 3 and 4 . As the percentage of benzo(a)pyrene is often used to characterise mixtures of PAHs, the ratio between the concentrations of pyrene and benz(a) pyrene have also been included. It seems that the actual exposure has been rather low in most studies. Table 4 summarises published data on 1-hydroxypyrene concentrations measured in patients and industrial populations and it is obvious that in these studies the actual exposures to PAHs were much higher. Nevertheless, during some operations such as the inspection of furnaces (study 2),

Table 7 Urinary 1-hydroxypyrene concentrations ( $\mu \mathrm{g} / \mathrm{g}$ creatinine) in workers at the needle coke plant

\begin{tabular}{|c|c|c|c|c|}
\hline & \multicolumn{2}{|l|}{ Maintenance } & \multicolumn{2}{|l|}{ Operation } \\
\hline & Pre-shift & Post-shift & Pre-shift & Post-shift \\
\hline $\begin{array}{l}\text { Mean (SD) } \\
\text { Median } \\
\text { Range } \\
\mathrm{n}^{\star}\end{array}$ & $\begin{array}{l}1.09(0.68) \\
1 \cdot 12 \\
0 \cdot 16-2 \cdot 66 \\
13(44)\end{array}$ & $\begin{array}{l}3.52(2 \cdot 78) \\
1.97 \\
0.32-10 \cdot 68 \\
13(58)\end{array}$ & $\begin{array}{l}1.25(0.86) \\
1.09 \\
0.28-3.04 \\
12(38)\end{array}$ & $\begin{array}{l}3.95(6.57) \\
2.20 \\
0.43-25.53 \\
12(52)\end{array}$ \\
\hline
\end{tabular}

${ }^{\star}$ Number of workers; number of assays in in parentheses: data are calculated from values averaged per person. replacement of burners (study 9), needle coke manufacture (studies 11a-c), and digging in soil contaminated with PAHs (study 13a), individual workers had been exposed to higher concentrations of PAHs than other petrochemical workers. In study $12 \mathrm{c}$ much higher results were found than in previous studies under identical circumstances (studies $12 a$ and $b$ ). This was due to one laboratory worker who always had raised urinary 1hydroxypyrene concentrations (study 12d) that could not be explained by occupational exposure.

NEEDLE COKE PLANT STUDY

Exposure to PAHs during needle coke

manufacture

Table 5 shows the results of air sampling. Higher PAH concentrations have been measured with static sampling than with personal air monitoring. The relative amount of pyrene as a percentage of the benzene soluble matter ranged from $<2 \%$ to $9 \cdot 2 \%$ (median $5.1 \%$ ) in the five static air monitoring samples. The values from the seven personal air samples had a median of $11.3 \%$ (range $2 \cdot 4 \%-18 \cdot 1 \%)$. The relative amounts of the various PAHs as assessed by air monitoring differ widely from the relative amounts as present in solid samples of the coke produced and the residues found on the litters of the coke chambers (table 6). Therefore, dermal exposure cannot simply be compared with inhalatory exposure.

Table 7 summarises the results of measurement of urinary 1-hydroxypyrene. The 1hydroxypyrene concentrations before a shift were about the same for operators (median $1.1 \mu \mathrm{g} / \mathrm{g}$ creatinine) and maintenance workers (median $1.2 \mu \mathrm{g} / \mathrm{g}$ creatinine) as were the concentrations after the shift (median values of $2 \cdot 2$ and $2.0 \mu \mathrm{g} / \mathrm{g}$ creatinine respectively).

The median urinary elimination rate constant $k_{e}$ of 1-hydroxypyrene was 0.052 $h^{-1}$, which corresponds to an apparent elimination half life of 13.4 hours (mean 10.4 (range 3.9-26.7) hours). There was no significant difference $(p=0 \cdot 190$; two tailed Student's $t$ test for unpaired data) in the mean (range) apparent half life of smokers (13.1 (7.5-25.4) hours; $\mathrm{n}=8$ ) and nonsmokers (8.5 (3.9-26.7) hours; $n=7)$, nor was there such a difference $(p=0.790)$ in half life between operators $(11.0(3.9-26.7)$ hours; $\mathrm{n}=7$ ) and maintenance workers $(10 \cdot 0(6 \cdot 5-21 \cdot 8)$ hours; $n=9)$.

\section{Evaluation of routes of exposure}

The work registration data contained information on three potential methods of exposure to PAHs: smoking behaviour, potential inhalatory exposure (described in terms of the degree of steam formation and the temperature of the steam released), and potential der$\mathrm{mal}$ exposure (described in terms of the amount of coke remaining on the litters of the coke chambers). Multiple linear regression analysis of the 1-hydroxypyrene excretion data (after logarithmic transformation) was performed to estimate the relative contributions of dermal and inhalatory exposure to 
Table 8 Needle coke plant study: multiple regression analysis on the 1-hydroxypyrene concentration in post-shift samples as a function of the 1-hydroxypyrene concentration in the pre-shift samples, smoking behaviour, potential dermal exposure, and potential inhalatory exposure

\begin{tabular}{|c|c|c|c|c|}
\hline Population & Determinant & $\begin{array}{l}\text { Regression } \\
\text { coefficient } \\
(S E)\end{array}$ & p Value & $R^{2}$ value \\
\hline Total $(n=82)$ & $\begin{array}{l}\text { Intercept } \\
\text { Pre-shift } \\
\text { Non-smoking } \\
\text { Dermal exposure } \\
\text { Inhalatory exposure }\end{array}$ & $\begin{array}{r}-0.538(0.146) \\
0.440(0.082) \\
-0.305(0.152) \\
0.124(0.057) \\
0.242(0.122)\end{array}$ & $\begin{array}{l}0.0004 \\
0.0001 \\
0.049 \\
0.034 \\
0.050\end{array}$ & 0.448 \\
\hline Operators $(n=44)$ & $\begin{array}{l}\text { Intercept } \\
\text { Pre-shift } \\
\text { Non-smoking } \\
\text { Dermal exposure } \\
\text { Inhalatory exposure }\end{array}$ & $\begin{array}{r}0.378(0.199) \\
0.382(0.105) \\
-0.222(0.215) \\
0.081(0.080) \\
0.329(0.175)\end{array}$ & $\begin{array}{l}0 \cdot 066 \\
0.0008 \\
0.308 \\
0 \cdot 318 \\
0.067\end{array}$ & 0.424 \\
\hline $\begin{array}{l}\text { Maintenance } \\
\quad \text { personnel }(n=38)\end{array}$ & $\begin{array}{l}\text { Intercept } \\
\text { Pre-shift } \\
\text { Non-smoking } \\
\text { Dermal exposure } \\
\text { Inhalatory exposure }\end{array}$ & $\begin{array}{r}0.804(0.222) \\
0.542(0.134) \\
-0.476(0.228) \\
0.196(0.085) \\
0.097(0.174)\end{array}$ & $\begin{array}{l}0.001 \\
0.0003 \\
0.044 \\
0.028 \\
0.581\end{array}$ & 0.521 \\
\hline
\end{tabular}

$\mathrm{SE}=$ standard error of the estimate; $n=$ number of samples.

actual PAH uptake (table 8). In the total population of the needle coke plant, smoking, dermal, and inhalatory exposure are significant determinants and the regression coefficient associated with inhalatory exposure is about twice the coefficient associated with dermal exposure. If the analysis is carried out for operators and the group of maintenance workers separately, however, it seems that in operators the regression coefficient associated with inhalatory exposure is about four times larger than that associated with dermal exposure, whereas in maintenance personnel the regression coefficients associated with dermal exposure is almost twice that associated with inhalatory exposure to PAHs. This means that in maintenance personnel the dermal exposure is relatively more important than the inhalatory exposure compared with the operators.

\section{Discussion}

Any process in which organic material is burned with insufficient oxygen results in the formation of PAHs. As a consequence PAHs are normally present in the environment and people that are not occupationally exposed to PAHs excrete small amounts of 1-hydroxypyrene into the urine. Table 2 lists urinary 1-hydroxypyrene concentrations found by other investigators in persons not occupationally exposed to PAHs. We found a median 1-hydroxypyrene baseline concentration of $0.21 \mu \mathrm{g} / \mathrm{g}$ creatinine in 121 industrial workers (both smokers and non-smokers). The upper normal value for industrial workers, defined as the upper limit of the $95 \% \mathrm{CI}$ in these workers, was $1.0 \mu \mathrm{g} / \mathrm{g}$ creatinine. These values are somewhat lower than reported for Dutch control populations, also comprising both smokers and non-smokers, where a median value of about $0.5 \mu \mathrm{g} / \mathrm{g}$ creatinine was found, with a $95 \%$ percentile of $2 \cdot 1 \mu \mathrm{g} / \mathrm{g}$ creatinine. 1521 The background concentrations differ due to variations in the environmental PAH background in the different countries. In several polluted industrialised areas in China much higher background con- centrations were measured. ${ }^{1322}$ In general, smoking is not a confounding factor in studies with low occupational or non-occupational exposure 10112022 unless the study group contains heavy smokers. ${ }^{1216}$ In our studies smoking behaviour was not taken into account because the exposures were lower than reported for occupationally exposed workers in the other studies mentioned. An exception was made for the needle coke plant study. In this study smoking proved to be a significant determinant of 1-hydroxypyrene excretion in the maintenance workers (table 8).

In most of our studies during operations with potential exposure to PAHs, the values of urinary 1-hydroxypyrene (table 3) are in the same range as for non-occupationally exposed Dutch people (table 2) and do not differ widely from the exposure due to the normal background. This suggests that actual exposures to PAHs in our operations (table 3) are low compared with other published industrial activities with potential exposure to PAHs (table 4). Handling of materials containing PAH such as bitumens, aromatic extracts and slurry oils (studies 3, 6, 10, 12a, and $b$ ), did not lead to a significant increase in urinary 1-hydroxypyrene compared with the overall baseline value (table 1), although only dermal protection was (occasionally) used. During cleaning operations of several installations containing residues with high PAH content such as platforming, hydroconverter, desulphurisation installations, and steam boilers (studies 1, 4, 5, and 8), and during tank cleaning (study 7) the potential exposure to PAHs is much higher. Nevertheless, only small increases in urinary 1-hydroxypyrene were measured indicating that the personal protection used was mostly adequate. Individual values, however, were sometimes clearly raised (table 3). During some operations such as the replacement of burners in boilers and inspection of a furnace (studies 2 and 9), a substantial increase in median urinary 1-hydroxypyrene concentrations was found and most operators had 1hydroxypyrene values far outside the normal range. Also high 1-hydroxypyrene concentrations were measured in individual workers during digging in soil contaminated with PAHs (study 13a). Most of these exceptions were measured in 1987 (studies 2, 9, and 13a) when the biological monitoring programme was started and were related to specific, short term operations such as handling of heavily contaminated equipment. Since then, stricter use of personal protection devices was imposed on the operators. These measures were successful as during several operations with a higher potential exposure to PAHs (for example, studies 4, 5, and 8) much smaller actual exposures were measured. The difference was particularly evident in workers digging soils contaminated with PAHs, where after a more stringent application of dermal protection (impermeable PVC suits) a substantial decrease in urinary 1hydroxypyrene concentrations was found in 
1991 and 1992 (studies $13 \mathrm{~b}$ and c) compared with 1987 and 1988 (study 13a).

Notable exceptions, however, were the operators of the needle coke plant who had a continuous potential occupational exposure to PAHs. In the first survey at this plant in 1988 (study 12a) it was found that during normal operation $88 \%$ of the maintenance workers and $62 \%$ of the operators had urinary 1-hydroxypyrene concentrations above the upper normal value. The median value in maintenance operators was $2.8 \mu \mathrm{g} / \mathrm{g}$ creatinine, which is about 13 times the median value found in the baseline studies. Specific measures to control the exposure to PAHs resulted in a decrease in median urinary 1hydroxypyrene concentrations, but in 1990 (study 12b) $76 \%$ of the maintenance workers and $72 \%$ of the operators still had urinary concentrations greater than the upper normal value. To get a better insight into the cause of exposure, a more detailed study was performed in 1990 (the needle coke plant study). The details of this study allowed qualitative assessment of the contributions of inhalatory and dermal exposures. As expected dermal absorption contributed significantly to the excretion of 1-hydroxypyrene (table 8). It also seemed that dermal exposure was more important in maintenance workers than in operators. This could be expected as maintenance workers had a greater risk of skin contact than operators, who were more likely to be exposed to airborne PAHs. The data from the analyses of the air samples (table 5) and the coke residue (table 6) suggest that the ratio of carcinogenic PAHs to pyrene is much higher in the solid samples (dermal exposure) than in the air samples (inhalatory exposure). This implies that the same urinary 1-hydroxypyrene excretion may indicate a higher actual exposure to carcinogenic $\mathrm{PAH}$ in maintenance personnel than in operators.

The measurement of urinary 1-hydroxypyrene does not yet permit a valid assessment of the potential risk of cancer due to exposure to PAH. Although good correlations were found between 1-hydroxypyrene and airborne PAH concentrations, ${ }^{91013}$ and also between 1-hydroxypyrene and dermal exposure to $\mathrm{PAH},{ }^{15} 18$ these correlations in fact merely indicate that the ratio between pyrene and one or more (carcinogenic) PAHs is fairly constant for a specific process during the time span of the study. These ratios, however, as well as the ratios between the various carcinogenic PAHs may vary considerably from one process to another and even for specific processes, such as the production of coke, from one batch to another (tables 3 and 4). As a consequence, ratios between the concentrations of pyrene and benzo(a)pyrene, although often used, ${ }^{131823}$ are unsuitable for reliable assessment of risk. As dermal absorption of PAHs also seems to be of importance a valid evaluation of risk requires that the composition of the sources for both inhalatory and dermal exposure are known as well as the relative contribution from each of these routes of exposure.
The excretion data that were obtained in the needle coke plant study allow some calculations on the toxicokinetics of 1-hydroxypyrene. Apparent urinary excretion half lives were calculated for 16 persons and a considerable interindividual variation was found, values ranging from 3.9 to 26.7 hours with a mean value of 10.4 hours. There was no significant difference between the apparent half lives for smokers and non-smokers nor for operators and maintenance workers. In a combined dermal and inhalatory exposure to PAHs in coke oven workers half lives ranged from 6 to 35 hours. ${ }^{12}$ Our value fits nicely into this range, but is slightly smaller than the value for the median apparent half life for the urinary excretion of 1-hydroxypyrene of $16 \cdot 8$ (range 13.4 to $26 \cdot 3$ ) hours that was recently reported on combined exposure for workers employed in a graphite electrode and coke production plant. ${ }^{10}$ In oral intake a mean half life for the urinary excretion of 1-hydroxypyrene of only 4.4 (range $3 \cdot 1$ to $5 \cdot 9$ ) hours was found. ${ }^{23}$ The differences in the apparent half lives may be due to slow dermal absorption or delayed release from pyrene stored in adipose tissue limiting the excretion rate. The wide variation in half life may also be explained by these effects. A moderately fast elimination phase, followed by a more prolonged phase with a half life of several days was reported in creosote workers. ${ }^{19}$ This slow elimination phase suggests that pyrene accumulates in a secondary compartment, most probably adipose tissue, from which it is only slowly released. This means that if someone is subjected to continuous occupational exposure to PAHs his baseline value should gradually rise above the normal background value until an equilibrium between uptake and elimination is reached. Such a rise was indeed found in the baseline values of paving workers, coke oven workers and aluminum smelters (table 1). We performed baseline studies in six different settings that could potentially cause exposure to PAHs. These groups included laboratory workers handling oils containing PAHs, people handling bitumens, ground workers, and boiler operators. An increase in 1-hydroxypyrene baseline values was not found in any of these groups indicating that exposure is so low that accumulation does not occur (table 2).

Our studies confirm the suitability of urinary 1-hydroxypyrene as a biomarker for the assessment of exposure to PAHs. It seems that in the petrochemical industry actual exposure to PAHs as measured by urinary 1-hydroxypyrene is low in many different operations with potential exposure to PAHs. This is partially due to the use of personal protective equipment in some situations. An exception was the workers involved in the production of needle coke where urinary 1hydroxypyrene concentrations were mostly greater than the upper normal value for nonoccupationally exposed persons. It was shown that in these workers dermal absorption may significantly contribute to the overall exposure to PAHs. 
We are indebted to Dr E A van der Meulen (Department of Epidemiology and Biostatistics; Erasmus University; Rotterdam; The Netherlands) for statistical advice. The tech-
nical assistance by C D van der Giessen (Department of nical assistance by C D van der Giessen (Department of B V; Moerdijk; The Netherlands) and $H$ van der Waal (Biomedical Laboratory, Shell Nederland Raffinaderij B V; Rotterdam; The Netherlands) is also gratefully acknowledged.

1 International Agency for Research on Cancer Monographs. Polynuclear aromatic compounds. Part 1: Chemical, environmental and experimental data. $L A R C$ Sci Publ 1983;32.

2 Boyland E, Sims P. Metabolism of polycyclic compounds-23. The metabolism of pyrene in rats and pounds-23. The metabolism of

3 Keimig SD, Kirby KW, Morgan DP, Keiser JE, Hubert TD. Identification of 1-hydroxypyrene as a major metabolite of pyrene in pig urine. Xenobiotica 1983;13: 415-20.

4 Jongeneelen FJ, van den Akker W, Bos RP, Anzion RBM, Theuws JLG, Roelofs HMJ, Henderson PT. 1Hydroxypyrene as an indicator of the mutagenicity of coal tar after activation with human liver preparations. Mutat Res 1988;204:195-201.

5 Zhao Z, Quan W-Y. Identification of 1-hydroxypyrene in urine of dogs after pyrene injection and its excretion. foumal of Environmental Science (China) 1992:3:127-32.

6 Jacob J, Brune H, Gettbarn G, Grimmer D, Heinrich U, Mohtashamipur et al. Urinary and faecal excretion of pyrene and hydroxypyrene by rats after oral, intraperipyrene and hydroxypyrene by rats after oral, intraperi-
toneal, intratracheal or intrapulmonary application. toneal, intratracheal or

7 Weyand EH, Wu Y, Patel S. Urinary excretion and DNA binding of coal tar components in B6C3F1 mice following ingestion. Chemical Research in Toxicology 1991;4 466-73.

8 Brandt HCA, De Groot PC, Molyneux MKB, Tindle PE. Sampling and analysis of bitumen fumes. Ann Occup Hyg 1985;29:27-80.

9 Tolos WP, Shaw PB, Lowry LK, MacKenzie BA, Deng JF. 1-Pyrenol: a biomarker for occupational exposure to polycyclic aromatic hydrocarbons. Appl Occup Environ polycyclic aromatic
Hyg 1990;5:303-9.

10 Buchet JP, Gennart JP, Mercado-Calderon F, Delavignette JP, Cupers L, Lauwerys R. Evaluation of exposure to polycyclic aromatic hydrocarbons in a coke production and a graphite electrode manufacturing plant: assessment of urinary excretion of 1-hydroxypyrene as a biological indicator of exposure. $\mathrm{Br} \mathcal{F}$ Ind Med 1992;49:761-8.

11 Jongeneelen FJ, Anzion RBM, Leijdekkers C-M, Bos RP, Henderson PT. 1-Hydroxypyrene in human urine after exposure to coal tar and a coal tar derived product. Int Arch Occup Environ Health 1985;57:47-55.

12 Jongeneeup RBM, van der Loop F, Bos RP, van Veen HG. Ambient and biological monitoring of coke oven workers: determinants of the internal dose of polycyclic aromatic hydrocarbons. $\mathrm{Br} \mathcal{F}$ Ind Med 1990;47:454-61.

13 Zhao Z-H, Quan W-Y, Tian D-H. Urinary 1-hydroxypyrene as an indicator of human exposure to ambient polycyclic aromatic hydrocarbons in a coal-burning environment. Sci Total Environ 1990;92:145-54

14 Bos RP, Jongeneelen FJ. Nonselective and selective methods for biological monitoring of exposure to coal-tar products. LARC Sci Publ 1988;89:389-95.

15 Jongeneelen FJ, Bos RP, Anzion RBM, Theuws ILG, Henderson PT. Biological monitoring of polycyclic aromatic hydrocarbons-metabolites in urine. Scand $f$ matic hydrocarbons-metabolites in

16 Burgaz S, Borm PJA, Jongeneelen FJ. Evaluation of urinary excretion of 1-hydroxypyrene and thioethers in workers exposed to bitumen fumes. Int Arch Occup Environ Health 1992;63:397-401.

17 Clonfero E, Zordan M, Venier P, Paleologo M, Levis AG, Cottica D, et al. Biological monitoring of human exposure to coal tar-urinary excretion of total polycyclic aromatic hydrocarbons, 1-hydroxypyrene and mutagen in psoriatic patients. Int Arch Occup Environ Health 1989;61:363-8.

18 Jongeneelen FJ, Scheepers PTJ, Groenendijk A, van Aerts LAGJM, Anzion RBM, Bos RP, Veenstra SJ. Airborne concentrations, skin contamination and urinary metabolite excretion of polycyclic aromatic hydrocarbons lite excretion of polycyclic aromatic hydrocarbons among paving workers exposed to coal tar
tars. Am Ind Hyg Assoc $\mathcal{f} 1988 ; 49: 600-7$.

19 Jongeneelen FJ, Anzion RBM, Scheepers PTJ, Bos RP, Henderson PT, Nijenhuis EH, et al. 1-Hydroxypyrene in urine as a biological indicator of exposure to polycyclic aromatic hydrocarbons in several work environments. Ann Occup Hyg 1988;32:35-43.

20 Jongeneelen FJ, Bos RP, Henderson PT. Metabolites of polycyclic aromatic hydrocarbons in urine of exposed workers. Toxicol Environ Chem 1988;16:295-307.

21 Jongeneelen FJ, Anzion RBM, Henderson PT Determination of hydroxylated metabolites of polycyclic aromatic hydrocarbons in urine. $₹$ Chromatogr 1987; 413:227-232.

22 Zhao Z-H, Quan W-Y, Tian D-H. Experiments on the effects of several factors on the 1-hydroxypyrene level in human urine as an indicator of exposure to polycyclic aromatic hydrocarbons. Sci Total Environ 1990;113: 197-207.

23 Buckley TJ, Lioy PJ. An examination of the time course from human dietary exposure to polycyclic aromatic hydrocarbons to urinary elimination of 1-hydroxypyrene. Br F Ind Med 1992;49:113-24. 